\title{
Role of tumor-derived extracellular vesicles in cancer progression and their clinical applications (Review)
}

\author{
FUHAO QIAO $^{1}$, PENG PAN $^{2}$, JIAPING YAN $^{1}$, JING SUN $^{1}$, YAN ZONG $^{1}$, \\ ZHIYONG WU ${ }^{1}$, XIAOQIN LU ${ }^{1}$, NA CHEN ${ }^{1}$, RUI MI ${ }^{3}$, YONGBIN MA ${ }^{3}$ and YUAN JI ${ }^{2}$ \\ ${ }^{1}$ Department of Clinical Laboratory, Traditional Chinese Medicine Hospital of Xintai, Xintai, \\ Shandong 271200; ${ }^{2}$ School of Medicine, Soochow University, Suzhou, Jiangsu 215123; \\ ${ }^{3}$ School of Medicine, Jiangsu University, Zhenjiang, Jiangsu 212013, P.R. China
}

Received October 9, 2018; Accepted February 15, 2019

DOI: $10.3892 /$ ijo.2019.4745

\begin{abstract}
Extracellular vesicles (EVs), including micro-vesicles and exosomes, are heterogeneous small membranous vesicles shed from the surface of myriad cells and are crucial in mediating intercellular communication. The vertical trafficking of cargo to the plasma membrane and subsequent redistribution of surface lipids may contribute to EV formation. Tumor-derived extracellular vesicles (TD-EVs) can carry complex, bioactive cargo, such as nucleic acids and proteins, during tumor metastasis. Paracrine information gets relayed by TD-EVs to adjacent tumor cells and this allows a crosstalk between malignant cells. These structures may even move to a distant metastatic lesion and modulate the tumor microenvironment to form a premetastatic niche. Thus, TD-EVs might be potential biomarkers for tumor development and metastasis. Additionally, EVs are promising candidates for use as cell-free vaccines or as vehicles for the delivery of specific tumor therapeutic molecules. Genetically modified microvesicles and engineered exosomes have shed light on a novel strategy for tumor-targeted gene therapy. This review focuses on the role of EVs in tumor development and metastasis and their possible applications in the advanced diagnosis and therapy of cancer and personalized medicine.
\end{abstract}

\section{Contents}

1. Introduction

2. General characteristics of EVs and TD-EVs

3. TD-EVs associated with cancer

4. EVs applied for diagnosis and therapy of cancer

5. Conclusions and future direction

Correspondence to: Dr Fuhao Qiao, Department of Clinical Laboratory, Traditional Chinese Medicine Hospital of Xintai, 860 Qingyun Street, Xintai, Shandong 271200, P.R. China

E-mail: qiaotingshanvip@163.com

Key words: tumor-derived microvesicles, premetastatic niche, biomarkers, targeted therapy

\section{Introduction}

To date, it has been considerably appreciated that extracellular vesicles (EVs) are crucial for intercellular communication, although they have previously been viewed as mediating these functions through soluble factors (1). EVs are majorly classified into three classes: ectosomes or shedding microvesicles (MVs), exosomes, and apoptotic bodies (ABs), based on their sizes and origins. MVs are released from the plasma membrane instead of internal membranes (as is the case for exosomes) and contain different protein components. In addition, MVs are larger and more heterogeneous in size than exosomes, ranging from $100 \mathrm{~nm}$ to $1 \mu \mathrm{m}$, while exosomes are $40-100 \mathrm{~nm}$ (2). Although MVs are not exactly identical to exosomes, they share similar biological functions (3). This review majorly focuses on cargo sorting in EVs, in particular in MVs and exosomes. With small dimensions and indistinguishable structures, these shedding vesicles conveniently function as bioactive cargo in order to facilitate intercellular communication (4). Indeed, the biological functions of EVs are not restricted to cell communication, and may have several applications. In order to comprehensively understand EV functions, we identified the role of each EV protein cargo (reported data to date from the Vesiclepedia database; http://microvesicles.org/) in general biological processes, and the 10 primary biological activities were noted, as presented in Fig. 1 (5). Notably, the two major biological roles of $\mathrm{EV}$ protein contents were signal transduction and cell communication (22.5 and $21.1 \%$, respectively), accounting for $\sim 50 \%$ of the total EV proteins. The importance of EVs in cell-to-cell communication and signal transduction was reported recently $(6,7)$. These membrane-bound sacs are assembled, transported across lipid bilayer membranes, and eventually shed from the surface of their parent cells. Once formed, EVs are paracellularly or distantly transported to the target release sites and are internalized into the recipient cells. Their cargo content is further released and mediates various processes, including signal transduction, cell communication, and transport (8). Considering the heterogeneity of membrane-bound sacs, they may also contribute to other biological activities, such as metabolism, cell growth and adhesion $(9,10)$. 
EVs' abilities of mass transport and information exchange between the cells is generally owed to their deposited complex molecules, such as multiple types of DNA, mRNA, small noncoding RNA, and proteins (11). Membrane-bound sacs secreted from different cell sources vary greatly in the quantity, dimension, their deposition, and even surface markers; thus, heterogeneity is observed among these membranous structures (12). A high-resolution proteomic and lipidomic analysis of exosomes and MVs from various cell sources revealed that U87 exosomes were enriched in sphingomyelins, whereas Huh7 and MSC exosomes were specifically enriched in cardiolipins (13). In the present review article, four gene sets of $\mathrm{EV}$ cargo content from different cell sources (data from Vesiclepedia database to date; http://microvesicles.org/) were compared and the results are depicted in the Venn diagram of Fig. 2. As presented in Fig. 2, the gene expression profiles of the endothelial cells, epithelial cells, osteoblasts, and monocytes derived from extracellular vesicles (EN-EVs, EP-EVs, OS-EVs, and MO-EVs, respectively) differ markedly, despite some small numbers of overlapping genes. The larger portion of the EV cargo gene profile in each cell source completely differs from the other three sources, and the proportion of EN-EVs, EP-EVs, MO-EVs, and OS-EVs is 67.6, 16.1, 29.8 and 13.8\%, respectively. In specific, the noncoincident regions of the four cargo gene sets contain respective marker genes that represent their original tissue characteristics. For example, in the present analysis there are four genes that exist only in the osteoblasts, namely four and half LIM domains 2 (FHL2), alkaline phosphatase biomineralization associated (ALPL), acid phosphatase 1 (ACP1), and calcium voltage-gated channel auxiliary subunit $\alpha 2 \delta 1$ (CACNA2D1). Notably, both ALPL and ACP1 phosphatases are specific markers of osteogenic differentiation, and are crucial in the formation, development, and metabolism of bone tissues $(14,15)$. This indicates that EVs tend to load tissue-specific cargo content, which expresses similar characteristics with their parent cells.

Indeed, EVs generally carry a series of tissue-specific characteristics or 'labels' that reveal the secretory cells from which they have originated $(16,17)$. For example, exosomes derived from cardiomyocytes can be denoted as 'cardiosomes' and facilitate a series of metabolic processes in target cells (18). This is crucial for distinguishing between numerous types of EVs and further investigating their specific roles. To date, studies aimed at discovering such labels have majorly focused on the proteome or lipidome within EVs. A recent study revealed that the content of exosomes and MVs derived from various cell sources considerably differed from each other at the proteomic level, which might be explored as an additional 'vesiculome' biomarker. By contrast, it has been demonstrated that protein enrichment in the exosomes distinguished cancer cells from stem cells, suggesting that they might essentially become a useful general cancer marker (19). Hence, it can be concluded that there is a difference between the applications of exosomes and MVs: the biomarker value of exosomes might lie in indicating sorting dysregulation in their source cells, whereas that of MVs might lie in reflecting the content of their source cells.

In vivo experiments have also been performed in recent years in an effort to identify the different $\mathrm{MV}$ origins from certain cell type sources. For instance, labeling MVs/exosomes with fluorescent lipophilic dyes (DiI, DiO, DiD and DiR) or lipophilic radiotracer (99mTc-HMPAO) is an important way to track or monitor the movement of MV in vivo $(20,21)$. However, these methods are mainly based on the non-specific lipid compositions of the membrane structure of MV, and thus these dyes are currently unable to identify the specific origin of MVs from different cell types. In particular, a novel method integrated with ultrasmall Mn-magnetofunctionalized $\mathrm{Ag} 2 \mathrm{Se}$ quantum dots and excellent near-infrared fluorescence or magnetic resonance imaging capabilities has been developed for instant efficient labeling of MVs for their in vivo high-resolution dual-mode tracking (22). However, even this effective and sensitive tracking method is unable to recognize the derivation of MVs, since it is non-specific and labeling of MVs occurs in vitro. From a review of the recent literature, it appears that no effective means exist to date to determine which MVs come from which cell type in in vivo experiments, and further studies will be required to address this important issue.

In addition to cell sources, physiological and disease conditions can also affect the content of vesicle cargo (23). The ratio of specific/non-specific proteins in EVs changes under pathological state. For example, brain-specific proteins in MVs, such as myelin basic protein, proteins of coagulation cascade and focal adhesion, were upregulated, while non-specific proteins, such as albumin, were downregulated with adverse outcomes in lacunar infarction (24). In particular, the malignant transformation of normal cells generally affects EV secretion, leading to aberrant shedding and oncogenic nucleic acid and protein content enrichment (25). The selection of EVs is strictly regulated; nevertheless, this highly regulated process is frequently perturbed in tumors $(26,27)$.

The following sections of the present review will focus on recent reports on tumor derived EVs (TD-EVs) and consider their specific and complex characteristics. In addition, the recent literature on genetically modified MVs and engineered exosomes associated with the tumor-targeted gene therapy will be discussed.

\section{General characteristics of EVs and TD-EVs}

Collectively, EVs can be isolated and separated from culture supernatants and various body fluids, such as plasma, urine, lymph, tissue, and cerebrospinal fluid (28). Ultracentrifugation and immunomagnetic separation techniques are common methods for EV separation, and via high resolution microscopy, the secretion process of EVs as well as their size, morphology, and ultrastructures can be visually observed. Scanning electron microscopy was used by our group to observe the secretion and structure of human bone marrow mesenchymal stem cell (hBMSC)-derived MVs (29). The hBMSCs were isolated from three male patients with acute myeloid leukemia aged 31, 35 and 36, that were admitted to Traditional Chinese Medicine Hospital of Xintai (Xintai, China) from June 2018 to September 2018, with written informed consent. To observe the release process of MVs from the cell surface, hBMSC-MVs were imaged under a scanning electron microscope. As presented in Fig. 3, several membrane-bound sacs were observed being shed from the membrane of hBMSCs. These 


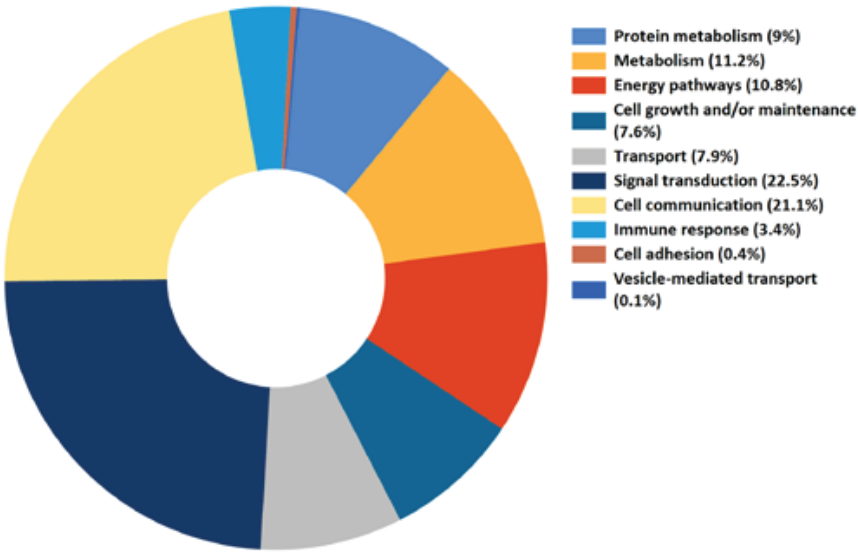

Figure 1. General biological processes of extracellular vesicle protein content identified in Vesiclepedia database. FunRich, a biological process enrichment analysis tool, was used to analyze the role of all database entries in the biological process and the 10 most common biological activities are presented in this figure.

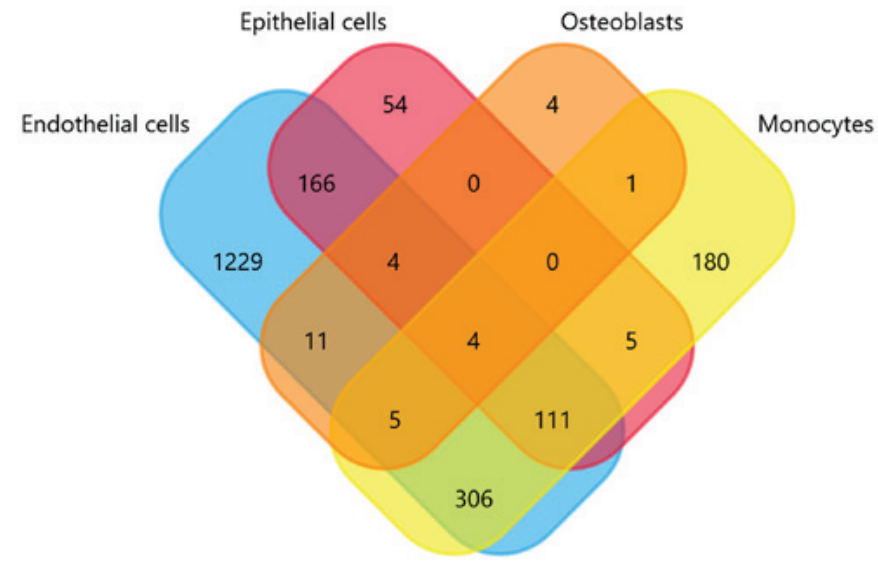

Figure 2. Extracellular vesicle gene cargo in Vesiclepedia database derived from four different cell sources (endothelial cells, epithelial cells, osteoblasts, and monocytes) is compared. FunRich, a gene enrichment analysis tool was used to indicate the similarities and differences of these four gene sets from distinct parent cell sources, and the results were presented in the Venn diagram.

were small, spheroidal, membranous structures ranging from 200 to $1,000 \mathrm{~nm}$ in size (exosomes range from 30 to $150 \mathrm{~nm}$ ).

In coordination with morphological identification, the molecular phenotype of EVs is generally detected via flow cytometric analysis; for example, CD9, CD81, CD82, CD122 and CD163, which are related to lipid microdomains, are relatively specific molecular markers expressed on the membrane surface of MVs. Two of these proteins, CD9 and CD63, are among the top 12 most commonly identified proteins in MVs (30). Therefore, they are commonly used for detection and immunopurification of MVs following isolation (31). In general, the protein cargo carried by EVs belongs to two categories: specific and nonspecific proteins. The latter majorly comprises proteins associated with biogenesis and common biological functions of EVs such as HSPs, TM4SF, metabolic enzymes, and cytoplasmic and signal transduction proteins (32). Specific proteins exist only in EVs derived from certain distinctive cell sources, which always carry their own 'labels', as aforementioned. These label

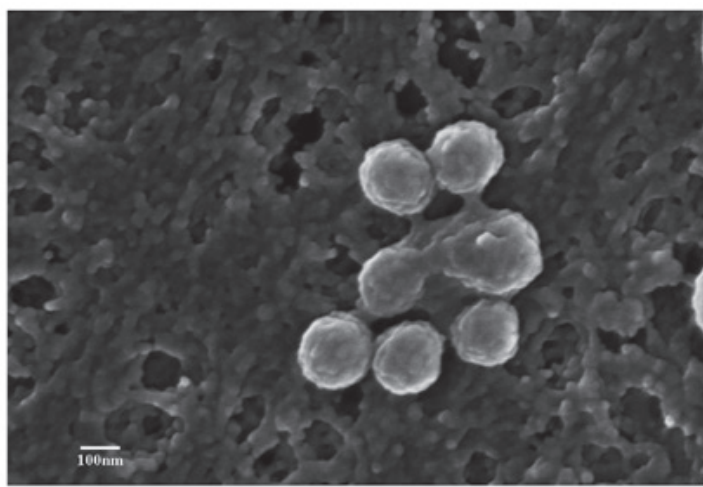

Figure 3. Structures on the surface of human mesenchymal stem cells were scanned via scanning electron microscopy. The spheroidal microvesicles were observed being shed from the cell membrane surface (Scale bar, $100 \mathrm{~nm}$ ).

proteins are closely associated with the function of releasing EVs, which also reflects the biological effect of their parent cell sources (19). In addition to protein cargo, nucleic acids, in particular noncoding RNA, have been reported to be crucial in biological functions of EVs (33). Collectively, all cargo content within EVs (proteins, nucleic acids or lipids) coordinate with each other to fulfill their own responsibilities.

Of note, TD-EVs have their own specific 'labels,' which distinguish them from the normal cell-derived EVs. Several studies have reported that TD-EVs can carry oncogenic membraneproteinsornucleicacidstofacilitatetumorprogression and a significant difference was observed in the proportion of oncogenic proteins and nucleic acids between normal and malignant cell derived EVs (34). For example, chromosome segregation 1 like (CSE1L), an important membrane protein carried by MVs, has been reported to mediate Ras-triggered MV generation and metastasis of B16F10 melanoma cells (35). Another study reported that endothelial cells developed chemoresistance upon uptake of transient receptor potential cation channel subfamily C member 5 (TrpC5) carried by adriamycin-resistant breast cancer cell-derived MVs (36). Thus, tumor cells are skilled in employing TD-EVs to spread oncogenic information throughout the body of an organism, facilitating the progression of cancer. We reviewed the recent literature regarding the roles of TD-EVs, and discovered that TD-EVs have been demonstrated to accelerate various aspects of cancer progression.

\section{TD-EVs associated with cancer}

Massive release of TD-EVs consecutive to cancer treatment. It has been reported that cancer therapies, such as photodynamic treatment, radiotherapy, or chemotherapy, trigger massive $\mathrm{EV}$ production, which is followed by release of oncogenes and oncoproteins (37). To evade chemotherapeutic agents, cancer cells can increase active drug efflux via EV shedding, and the enhanced level of TD-EVs has been identified as one of the most important mechanisms contributing to cancer chemotherapy resistance (38). Remarkably, it has been demonstrated that TD-EVs can cause the development of tumor cells with an acquired drug resistance phenotype and contribute to multidrug resistance. The release of multidrug resistance proteins, such as Pgp-1 and lipid ceramide derived 
from TD-MVs, was reported to mediate drug resistance (39). In addition, TD-EVs could also mediate chemoresistance by taking up chemotherapeutic drugs, and thus, limiting their bioavailability for treating cancer cells (40). Considering its multidrug resistant role, recent studies have been devoted to inhibiting the release of TD-EVs from tumor cells in order to sensitize them to chemotherapy. For instance, a study by Kholia et al (41) reported that inhibiting the release of TD-EVs from prostate cancer cells reduced the drug resistance of these cells upon methotrexate treatment. In addition to mediating drug resistance, the elevated levels of EVs in tumor patients have also been reported to influence multiple tumorigenic processes, including cell proliferation, epithelial-mesenchymal transition (EMT), angiogenesis, and premetastatic niche formation. Thus, the transport of pathological growth factor receptors, various soluble proteins, and miRNAs by TD-EVs aids tumor survival and spread.

TD-EVs transfer oncogenic cargo content to promote tumor progression. Recent studies emphasized on the role of TD-EVs in tumor colonization and progression by regulating tumor invasion, angiogenesis, and suppressing immunity (42). An important method of TD-EVs to facilitate tumor development and invasion is via transferring the oncogenic cargo content. It is undeniable that several other alternatives exist for tumor cell communication: direct cell-to-cell contact; paracrine soluble cytokines signaling to local cells; and cell-matrix interactions (43). Furthermore, another important way of cell communication occurs through the TD-EVs transferring their cargo to distant loci, a process that had been demonstrated to facilitate the premetastatic niche formation and tumor metastasis. The present review focuses on the role of TD-EVs in tumor proliferation, EMT, extracellular matrix remodeling, angiogenesis, and immunosuppression. Notably, TD-EVs were abundant in the noncoding and specific coding RNA; the elevated levels of amplified, missing, or mutated oncogene sequences and transposable elements, might confer oncogenic information in the recipient cells (44). It has been reported that gastric cancer-derived exosomes could promote tumor proliferation via activating the PI3K/AKT and MAPK/ERK signaling pathways (45). It is well-known that EMT, one of the hallmarks of aggressive cancer, confers tumors with a more malignant and dedifferentiated phenotype, because of a conversion from motionless epithelial cells into a more active and motile cell type, mesenchymal cells (46). Previous studies have reported that TD-EVs carried the full-length tissue factor (fITF) III or CD142 and induced the mesenchymal phenotype expression in tumors (47). Transforming growth factor- $\beta$ is a key regulator responsible for EMT, which contributes to the migration and spread of the tumor. Kim et al (48) reported that TD-MVs' content cargo (enriched in miR-23a) induced EMT in human lung adenocarcinoma A549 cells via TGF- $\beta 1$ signaling, suggesting that the tumor could facilitate its invasion and malignant progression in an autocrine way by releasing EVs.

The famous hypothesis 'seed and soil' put forward by Stephen Paget in 1889 was a milestone for the study of tumor metastasis and remains current even today (49). This hypothesis signifies the importance of the microenvironment at the distant site (soil), not simply focusing on the tumor itself (seed). Tumor microenvironment (TME) is the foundation of tumor development and is necessary for malignant cell survival (50). It comprises of cellular [such as inflammatory cells, immunocytes, endotheliocytes, mesenchymal stem cells (MSCs), and cancer-associated fibroblasts (CAFs)] and noncellular components (such as cytokines, chemokines, and matrix proteins). These two components coordinate together to form the whole complex TME and to support the survival and growth of tumors (51). Extracellular matrix (ECM), predominantly comprised of glycoproteins, is the scaffold including all the materials surrounding the cells, except from lymph and blood, and is an essential component of the TME (52). TD-EVs shuttle across this scaffold and construct a bridge between the tumor cells and surrounding stromal cells to promote the spread of tumors. The degradation of ECM further releases growth factors, enhancing the migration and invasion capacity of the malignant cells (53). In addition, it has been reported that the TME facilitates the differentiation of fibroblasts to myofibroblasts or CAFs, which subsequently secrete matrix metalloproteinases, further degrading the matrix proteins and remodeling the ECM (54). For example, Cho et al (55) reported that breast cancer-derived exosomes could convert the adipose derived mesenchymal stem cells into tumor-associated myofibroblast-like cells, which contributed to the progression and malignancy of tumors (55). Collectively, TD-EVs appear to be crucial in ECM remodeling and as a result in tumor migration and invasion (Fig. 4).

Furthermore, increasing evidence suggests that TD-EVs promote tumor angiogenesis and repress the antitumor immune response, in order to protect the tumor cells from a hostile environment (56). The exact mechanisms for these effects of TD-EVs, however, remain unclear. Tumor-associated macrophages (TAMs) generally adopt an M2-like phenotype in tumors and the M2 polarization of TAMs has been regarded as one of most important hallmarks of cancer progression and metastasis (57). It has been reported that TD-EVs could deliver cargo content to facilitate the polarization of TAMs (58). For example, Ying et al (59) reported that epithelial ovarian cancer-derived exosomes induce polarization of TAMs via transport of miR222-3p, which activates the suppressor of cytokine signaling 3 (SOCS3)/signal transducer and activator of transcription 3 (STAT3) signaling pathway. Despite this, the delivery of miR-21 could also promote M2-like polarization of TAMs in snail-overexpressing cancer cells (60). Essentially, TAMs further secrete various angiogenic growth factors, such as vascular endothelial growth factor (VEGF), interleukin (IL)-6, granulocyte-colony stimulating factor (G-CSF), and tumor necrosis factor $\alpha$ (TNF- $\alpha)$, to induce formation of new vessels within the tumor tissues, which is essential for tumor cell survival under hypoxia conditions (61). Furthermore, TD-EVs were reported to repress the antitumor immune response by activating regulatory $\mathrm{T}$ cells and myeloid-derived suppressor cells (MDSCs), which further inhibit the targeted immune effect mediated by $\mathrm{CD}^{+} \mathrm{T}$ cells (62). Finally, the expression of Fas ligand (FasL) and TNF-related apoptosis-inducing ligand (TRAIL) on the surface of TD-EVs could also induce apoptosis of the cytotoxic $\mathrm{CD}^{+} \mathrm{T}$ cells, potentially enhancing tumor immune evasion.

TD-EVs facilitate premetastatic niche formation and tumor microenvironment remodeling. Tumor cells can communicate 

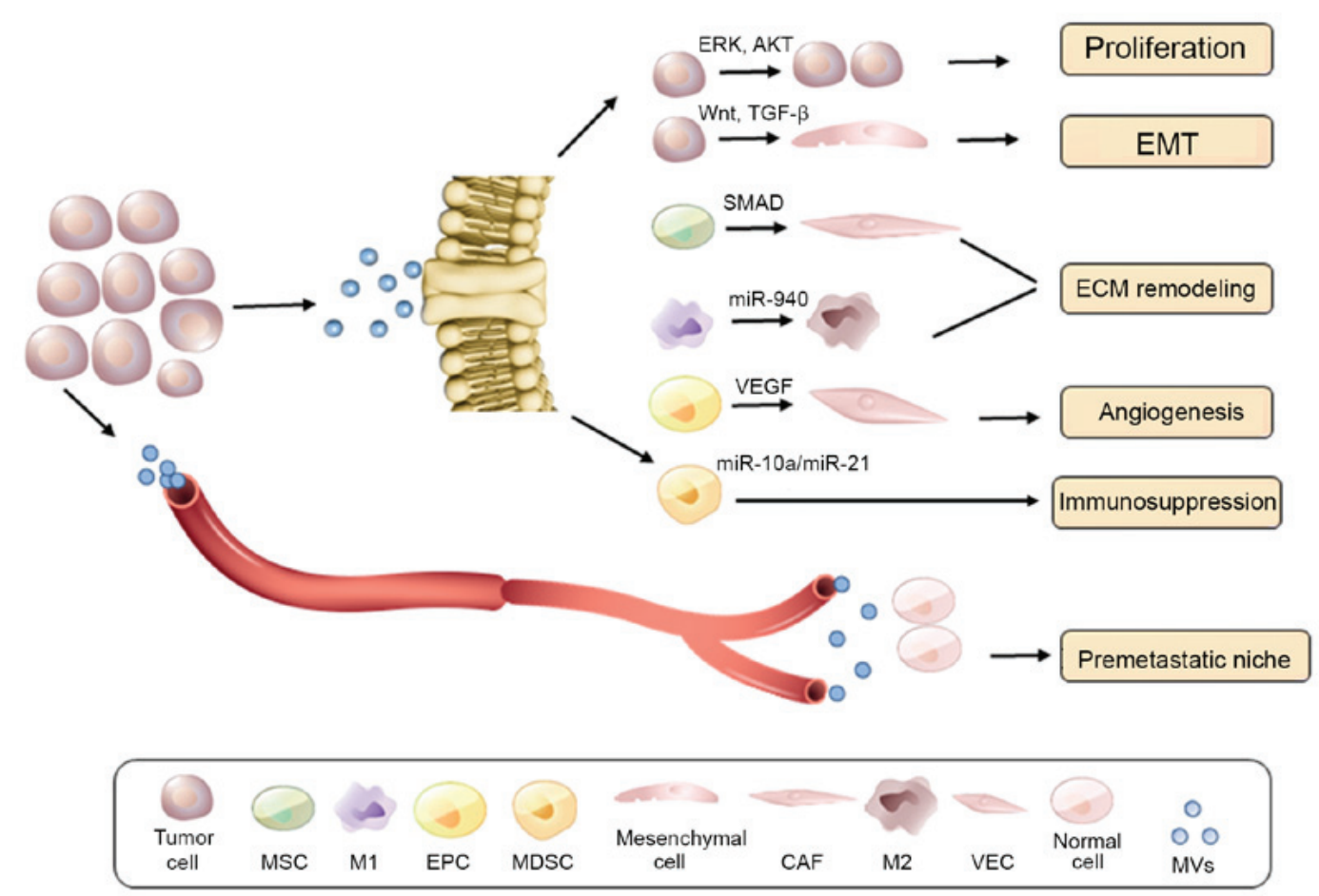

Figure 4. TD-EV mediated tumor progression and premetastatic niche education. At the primary tumor site, shed TD-EVs can promote tumor proliferation, EMT, ECM remodeling, angiogenesis, and potentially immunosuppression, via its oncogenic cargo content release. Furthermore, TD-EVs contribute to the distant early metastatic niche formation, which facilitates cancer metastasis. TD-EV, tumor-derived extracellular vesicle; EMT, epithelial-mesenchymal transition; ECM, extracellular matrix; MSC, mesenchymal stem cell; EPC, epithelial progenitor cell; MDSC, myeloid-derived suppressor cell; CAF, cancer-associated fibroblast; VEC, vascular endothelial cell; MVs, microvesicles; ERK, extracellular signal-regulated kinase; AKT, AKT serine/threonine kinase; TGF, transforming growth factor; VEGF, vascular endothelial growth factor.

with the surrounding tumor and stromal cells via the transport of cargo through TD-EVs; this facilitates tumor invasion, angiogenesis, and immunity suppression (63). Alternatively, TD-EVs also contribute to the formation of a premetastatic niche for the distant metastasis of cancer (64). Although the exact mechanism is not clear, TD-EVs can induce vascular leakage and interact with cells residing in remote organs. This interaction is selective and highly-specific, because TD-EVs have been demonstrated to be recruited to particular cell types located in specific organs, depending on their membrane protein composition (65). For instance, tumor exosome integrins have been reported to be involved in organotropic metastasis: integrin avb5 is associated with hepatic metastases, whereas $\alpha 6 \mathrm{~b} 4$ and $\alpha 6 \mathrm{~b} 1$ are related to lung metastases (66). Upon endocytosis by recipient cells, TD-EVs induce the expression of multiple inflammatory factors, such as S100, IL-8, IL-6, TGF- $\beta$ and TNF- $\alpha$, which contribute to the activation of stromal cells and remodeling of the ECM (67).

Stromal cells, ECM, inflammatory immune cells, and TD-EVs collectively form a permissive and attractive environmentforthemetastatic cells, alsocalled the premetastatic niche (PMN). Presumably, this early metastatic niche has been engineered and educated by TD-EVs to be prepared for metastatic tumor cell implantation (68). Hood et al (69) reported that melanoma cells preferred migrating to places with abundant TD-MVs. Melo et al (70) demonstrated that glypican-1 on the membrane of MVs released in body fluids of patients with cancer was considerably overexpressed and promoted proliferation and metastasis in cancer cells (70). Based on these evidences, TD-EVs are thought to be released to the perivascular space and easily get across into vessels or lymph capillaries (several $\mathrm{nm}$ in size) and the release of their cargo content (always abundant in oncogenic nucleic acids and proteins) at a distant site then contributes to the engineering of the PMN. This hypothesis might provide an explanation for the potential mechanism of tumor metastasis.

Education of PMN by TD-EVs might involve various aspects. For example, hypoxic TME notably contributes to an aggressive phenotype and drug resistance that leads to tumor progression, recurrence, and metastasis; however, the mechanisms underlying these processes remain unclear (71). Recently, it was reported that TD-EVs could be recruited and forced to arrive at the metastatic locus, which was conducive to the improvement of tumor oxygen levels, angiogenesis and metastasis (72). This might be one of the major causes of hypoxia in the tumor microenvironment. Furthermore, another important method through which TD-EV mediates the engineering of early metastatic niches is by participating in the formation of new blood vessels (73). Tumors commonly release a myriad of growth factors to induce blood vessel formation (angiogenesis), for providing oxygen and other essential nutrients through a dedicated blood supply to the tumor. It has been reported that chronic myeloid leukemia-derived exosomes induce angiogenic activity in HUVEC cells and promote angiogenesis in a Src-dependent manner (74). Collectively, through the education or engineering of the PMN by TD-EVs, tumor cells that have invaded and disseminated from an established primary tumor, are led to a distant locus via blood vessels to implant and grow into a secondary tumor (Fig. 4). 


\section{EVs applied for diagnosis and therapy of cancer}

Diagnostic role of EVs in cancer. Several studies have reported the significance of TD-EVs in tumor progression. Nevertheless, research on the application of TD-MVs for cancer diagnosis and therapy is relative scarce, and yet not well-rounded. The present article reviewed the recent literature regarding the TD-EV application for tumor diagnosis and therapy. To seek personalized precision healthcare, recent studies have emphasized the diagnostic role of EVs in cancer (75). With the rise of liquid biopsy tests and circulating tumor DNA analysis, scientists have been focusing on EV diagnostic technologies comprising total nucleic acid co-isolation [exosomal RNA (exoRNA) and circulating tumor DNA (ctDNA)], target capture, and bioinformatics (76). As MVs or exosomes can be detected in various body fluids, including saliva, urine and blood, EV diagnostics have been one of the most important components in liquid biopsy tests in addition to ctDNA and circulating tumor cells (CTCs). In addition, tests on circulating EVs might be a more precise and sensitive method for diagnosing cancer compared with CTCs, due to the high variability in the number of CTCs among different cancer patients (77). It has been reported that an apparent increase of MV concentration in the peripheral circulation of ovarian, breast, and pancreatic cancer is observed (78). Therefore, the concentrations of EVs could be measured in the body fluids of patients for an early cancer diagnosis. Another important part of EV diagnostics includes testing the cargo content, such as proteins and nucleic acids. Considering the common characteristics of EVs due to similar biogenesis and the overlap in its cargo content, they can be isolated and identified via general biomarkers regardless of their tissue of origin (79). Nevertheless, as aforementioned, EVs commonly carry a series of tissue-specific characteristics or 'labels' similar to their secretory cells in order to reveal their origin. Therefore, this property provides an identifiable and unique biosignature for individual cancer patients (80).

In particular, TD-EVs considerably differ from the common EVs in terms of cargo content, owing to their cancer promoting properties (81). For example, the expression of miR-21 in MVs derived from patients with esophagus squamous cell carcinoma was considerably higher compared with normal individuals, and this was closely associated with the progression and malignancy of esophageal carcinoma (82). Additionally, miR-141 derived from the serum of prostate cancer patients was overexpressed in the primary and secondary tumors (83). Whole-genome sequencing analysis revealed that MVs in sera from patients with pancreatic cancer covered the genomic dsDNA in all 24 human chromosomes (84). In addition, the genetic mutations associated with pancreatic ductal adenocarcinoma could be detected in these TD-MVs (85). These evidences indicate that the results of whole-genome sequencing analysis in TD-EVs match the gene profile of primary and secondary tumors. Proteins in TD-EVs are also associated with tumor progression and have been previously discussed in detail.

Therapeutic role of EVs in cancer. Considering the facilitating role of TD-EVs in PMN formation and tumor microenvironment remodeling, they may serve in the future as a target for the clinical treatment of cancer (86). For example, inhibition of
EV release prevents invasion of the tumor cells to surrounding stromal cells, by inhibiting the interactions between them, thereby hindering cancer angiogenesis and metastasis (87). In addition, it has been reported in several studies that TD-EVs promote drug-resistance of tumor cells in chemotherapy in several ways (88). Reports indicated that TD-MVs mediated the transition of the tumor cells from sensitive to drug-resistant, and thus elimination of TD-MVs might contribute in improving drug sensitivity and effect of chemotherapy (89). In addition, Her-2 positive MVs could repress the antiproliferation effect of trastuzumab on breast cancer cells; therefore, it may be possible to improve response to trastuzumab in patients by removing Her-2 positive MVs from their tumors (90).

Recently, nanotechnology has been widely applied for EV detection, characterization, and especially engineering (91). The use of EVs as a drug delivery platform for nanomedicine applications has been previously emphasized (92). Tian et al (93) used modified exosomes as doxorubicin delivery vehicles to target $\alpha v$-integrin-positive tumor cells. Furthermore, Mizrak et al (94) reported that genetically modified MVs that carried suicide nucleic acids or proteins could induce the death of schwannoma tumor cells. Liposoluble chemotherapeutic drugs, such as paclitaxel and lomustine, are able to penetrate the blood brain barrier, thus leading to increased side effects. Application of exosome engineering for targeted therapy has a great advantage in reducing drug side effects, compared with conventional methods, owing to its lipid bilayer structure, which can enwrap the therapeutic drugs to form a hydrophobic structure (95). Collectively, genetically engineered EVs may prove to be extremely useful novel strategies for targeted therapy in cancer.

\section{Conclusions and future direction}

EVs are heterogeneous membrane-bound sacs with multiple biological functions, such as signal transduction and intracellular communication. The release of their cargo content, including nucleic acids, proteins and lipids, contributes to several physiological and pathological processes. EVs commonly carry a series of tissue-specific characteristics or 'labels' that match their secretory cells to reveal their origin, a process that provides an identifiable and unique biosignature for individual cancer patients (96). TD-EVs are involved in various processes of tumor progression, such as malignant proliferation, EMT, ECM remodeling, and angiogenesis. In addition, they are crucial for premetastatic niche formation and tumor microenvironment remodeling, processes that contribute to cancer metastasis. Tests employing circulating EVs might be a more accurate and sensitive way for diagnosis of cancer compared with CTCs, and they may provide an easily identifiable and unique biosignature for individual cancer patients. Finally, thanks to the development of nanotechnology, EVs have been used as a drug delivery vehicle for targeted tumor therapy.

Taken together, this review article provides a comprehensive understanding of the roles of EVs in cancer progression, and reveals the characteristics of EVs originated from different sources, especially from different cancer cells, which has not been reported to date. Furthermore, the latest progress in the potential clinical applications of EVs were discussed. Our 
knowledge of the basic structures, cargo content and biological roles of EVs have tremendously progressed; however, more achievement is expected. For example, little is known as to how TD-EVs are recruited to distant loci and connect with the surrounding stromal cells (97). In addition, although it is established that the signatures among EVs differ based on their tissue of origin, their biological functions still cannot be distinguished according to these signatures. Further research will fully elucidate the roles and functions of TD-EVs, with the hope that EV-based strategies will be beneficial in the future for personalized precision healthcare in patients with cancer.

\section{Acknowledgements}

Not applicable.

\section{Funding}

This study was supported by the Shandong Medical and Health Science and Technology Development Plan (grant no. 2018WS047).

\section{Availability of data and materials}

Not applicable.

\section{Authors' contributions}

FHQ, PP, YBM, YJ and RM were involved in the conception of the study subject. JPY, JS, YZ, ZYW, XQL and NC were involved in the study design and the acquisition of data. PP, FHQ and RM were involved in the writing of the article and revised this manuscript. All authors read and approved the final manuscript.

\section{Ethics approval and consent to participate}

Not applicable.

\section{Patient consent for publication}

Not applicable.

\section{Competing interests}

The authors declare that they have no competing interests

\section{References}

1. Lee TH, D'Asti E, Magnus N, Al-Nedawi K, Meehan B and Rak J: Microvesicles as mediators of intercellular communication in cancer--the emerging science of cellular 'debris'. Semin Immunopathol 33: 455-467, 2011.

2. Meckes DG Jr and Raab-Traub N: Microvesicles and viral infection. J Virol 85: 12844-12854, 2011.

3. Raposo G and Stoorvogel W: Extracellular vesicles: Exosomes, microvesicles, and friends. J Cell Biol 200: 373-383, 2013.

4. Barteneva NS, Maltsev N and Vorobjev IA: Microvesicles and intercellular communication in the context of parasitism. Front Cell Infect Microbiol 3: 49, 2013.

5. Kalra H, Simpson RJ, Ji H, Aikawa E, Altevogt P, Askenase P, Bond VC, Borràs FE, Breakefield X, Budnik V, et al: Vesiclepedia: A compendium for extracellular vesicles with continuous community annotation. PLoS Biol 10: e1001450, 2012.
6. Ratajczak J, Wysoczynski M, Hayek F, Janowska-Wieczorek A and Ratajczak MZ: Membrane-derived microvesicles: Important and underappreciated mediators of cell-to-cell communication. Leukemia 20: 1487-1495, 2006.

7. Martínez MC, Larbret F, Zobairi F, Coulombe J, Debili N, Vainchenker W, Ruat M and Freyssinet JM: Transfer of differentiation signal by membrane microvesicles harboring hedgehog morphogens. Blood 108: 3012-3020, 2006.

8. Akers JC, Gonda D, Kim R, Carter BS and Chen CC: Biogenesis of extracellular vesicles (EV): Exosomes, microvesicles, retrovirus-like vesicles, and apoptotic bodies. J Neurooncol 113: $1-11,2013$.

9. Lawson C, Vicencio JM, Yellon DM and Davidson SM: Microvesicles and exosomes: New players in metabolic and cardiovascular disease. J Endocrinol 228: R57-R71, 2016.

10. Jansa R, Sustar V, Frank M, Susanj P, Bester J, Mancek-Keber M, Krzan M and Iglic A: Number of microvesicles in peripheral blood and ability of plasma to induce adhesion between phospholipid membranes in 19 patients with gastrointestinal diseases. Blood Cells Mol Dis 41: 124-132, 2008.

11. Loyer X, Vion AC, Tedgui A and Boulanger CM: Microvesicles as cell-cell messengers in cardiovascular diseases. Circ Res 114: 345-353, 2014.

12. Sullivan R: Epididymosomes: A heterogeneous population of microvesicles with multiple functions in sperm maturation and storage. Asian J Androl 17: 726-729, 2015.

13. Didiot MC, Hall LM, Coles AH, Haraszti RA, Godinho BM, Chase K, Sapp E, Ly S, Alterman JF, Hassler MR, et al: Exosome-mediated Delivery of Hydrophobically Modified siRNA for Huntingtin mRNA Silencing. Mol Ther 24: 1836-1847, 2016.

14. Cao FY, Fan JX, Long Y, Zeng X and Zhang XZ: A smart fluorescence nanoprobe for the detection of cellular alkaline phosphatase activity and early osteogenic differentiation. Nanomedicine (Lond) 12: 1313-1322, 2016.

15. Lau KH and Baylink DJ: Osteoblastic tartrate-resistant acid phosphatase: Its potential role in the molecular mechanism of osteogenic action of fluoride. J Bone Miner Res 18: 1897-1900, 2003-1900.

16. Aliotta JM, Pereira M, Johnson KW, de Paz N, Dooner MS, Puente N, Ayala C, Brilliant K, Berz D and Lee D: Microvesicle entry into marrow cells mediates tissue-specific changes in mRNA by direct delivery of mRNA and induction of transcription. Exp Hematol 38: 233-245, 2010.

17. Collino F, Deregibus MC, Bruno S, Sterpone L, Aghemo G, Viltono L, Tetta C and Camussi G: Microvesicles derived from adult human bone marrow and tissue specific mesenchymal stem cells shuttle selected pattern of miRNAs. PLoS One 5: e11803, 2010.

18. Waldenström A, Gennebäck N, Hellman U and Ronquist G: Cardiomyocyte microvesicles contain DNA/RNA and convey biological messages to target cells. PLoS One 7: e34653, 2012.

19. Haraszti RA, Didiot MC, Sapp E, Leszyk J, Shaffer SA, Rockwell HE, Gao F, Narain NR, DiFiglia M, Kiebish MA, et al: High-resolution proteomic and lipidomic analysis of exosomes and microvesicles from different cell sources. J Extracell Vesicles 5: 32570, 2016.

20. Zheng T, Pu J, Chen Y, Mao Y, Guo Z, Pan H, Zhang L, Zhang H, Sun B and Zhang B: Plasma Exosomes Spread and Cluster Around $\beta$-Amyloid Plaques in an Animal Model of Alzheimer's Disease. Front Aging Neurosci 9: 12, 2017.

21. Hwang DW, Choi H, Jang SC, Yoo MY, Park JY, Choi NE, Oh HJ, Ha S, Lee YS, Jeong JM, et al: Noninvasive imaging of radiolabeled exosome-mimetic nanovesicle using (99m)Tc-HMPAO. Sci Rep 5: 15636, 2015

22. Zhao JY, Chen G, Gu YP, Cui R, Zhang ZL, Yu ZL, Tang B, Zhao YF and Pang DW: Ultrasmall Magnetically Engineered Ag2Se Quantum Dots for Instant Efficient Labeling and Whole-Body High-Resolution Multimodal Real-Time Tracking of Cell-Derived Microvesicles. J Am Chem Soc 138: 1893-1903, 2016.

23. Abels ER and Breakefield XO: Introduction to Extracellular Vesicles: Biogenesis, RNA Cargo Selection, Content, Release, and Uptake. Cell Mol Neurobiol 36: 301-312, 2016.

24. Datta A, Chen CP and Sze SK: Discovery of prognostic biomarker candidates of lacunar infarction by quantitative proteomics of microvesicles enriched plasma. PLoS One 9: e94663, 2014.

25. Tricarico C, Clancy J and D'Souza-Schorey C: Biology and biogenesis of shed microvesicles. Small GTPases 8: 220-232, 2017. 
26. Al-Nedawi K, Meehan B, Micallef J, Lhotak V, May L, Guha A and Rak J: Intercellular transfer of the oncogenic receptor EGFRvIII by microvesicles derived from tumour cells. Nat Cell Biol 10: 619-624, 2008

27. Principe S, Hui AB, Bruce J, Sinha A, Liu FF and Kislinger T: Tumor-derived exosomes and microvesicles in head and neck cancer: Implications for tumor biology and biomarker discovery. Proteomics 13: 1608-1623, 2013.

28. Lee K, Shao H, Weissleder R and Lee H: Acoustic purification of extracellular microvesicles. ACS Nano 9: 2321-2327, 2015.

29. Tickoo SK, Lee MW, Eble JN, Amin M, Christopherson T, Zarbo RJ and Amin MB: Ultrastructural observations on mitochondria and microvesicles in renal oncocytoma, chromophobe renal cell carcinoma, and eosinophilic variant of conventiona (clear cell) renal cell carcinoma. Am J Surg Pathol 24: 1247-1256, 2000.

30. Silverman JM and Reiner NE: Exosomes and other microvesicles in infection biology: Organelles with unanticipated phenotypes. Cell Microbiol 13: 1-9, 2011

31. Mathivanan S,Lim JW, Tauro BJ, Ji H, Moritz RL and Simpson RJ: Proteomics analysis of A33 immunoaffinity-purified exosomes released from the human colon tumor cell line LIM1215 reveals a tissue-specific protein signature. Mol Cell Proteomics 9: 197-208, 2010.

32. Wenzel D, Schauermann G, von Lüpke A and Hinz G: The cargo in vacuolar storage protein transport vesicles is stratified. Traffic 6: 45-55, 2005 .

33. Miranda KC, Bond DT, Levin JZ, Adiconis X, Sivachenko A, Russ C, Brown D, Nusbaum C and Russo LM: Massively paralle sequencing of human urinary exosome/microvesicle RNA reveals a predominance of non-coding RNA. PLoS One 9: e96094, 2014

34. Arendt BK, Walters DK, Wu X, Tschumper RC and Jelinek DF: Multiple myeloma dell-derived microvesicles are enriched in CD147 expression and enhance tumor cell proliferation. Oncotarget 5: 5686-5699, 2014.

35. Liao CF, Lin SH, Chen HC, Tai CJ, Chang CC, Li LT, Yeh CM, Yeh KT, Chen YC, Hsu TH, et al: CSE1L, a novel microvesicle membrane protein, mediates Ras-triggered microvesicle generation and metastasis of tumor cells. Mol Med 18: 1269-1280, 2012.

36. Dong Y, Pan Q, Jiang L, Chen Z, Zhang F, Liu Y, Xing H, Shi M, Li J, Li X, et al: Tumor endothelial expression of P-glycoprotein upon microvesicular transfer of TrpC5 derived from adriamycin-resistant breast cancer cells. Biochem Biophys Res Commun 446: 85-90, 2014.

37. Aubertin K, Silva AKA, Luciani N, Espinosa A, Djemat A, Charue D, Gallet F, Blanc-Brude O and Wilhelm C: Massive release of extracellular vesicles from cancer cells after photodynamic treatment or chemotherapy. Sci Rep 6: 35376, 2016.

38. Qiu J, Yang G, Feng M, Zheng S, Cao Z, You L, Zheng L, Zhang $T$ and Zhao Y: Extracellular vesicles as mediators of the progression and chemoresistance of pancreatic cancer and their potential clinical applications. Mol Cancer 17: 2, 2018.

39. Muralidharan-Chari V, Clancy JW, Sedgwick A and D'Souza-Schorey C: Microvesicles: Mediators of extracellular communication during cancer progression. J Cell Sci 123 $1603-1611,2010$

40. Wang X, Xu C, Hua Y, Sun L, Cheng K, Jia Z, Han Y, Dong J, $\mathrm{Cui} \mathrm{Y}$ and Yang Z: Exosomes play an important role in the process of psoralen reverse multidrug resistance of breast cancer. J Exp Clin Cancer Res 35: 186, 2016.

41. Kholia S, Jorfi S, Thompson PR, Causey CP, Nicholas AP, Inal JM and Lange S: A novel role for peptidylarginine deiminases in microvesicle release reveals therapeutic potential of PAD inhibition in sensitizing prostate cancer cells to chemotherapy. J Extracell Vesicles 4: 26192, 2015. [J].

42. Al-Nedawi K, Meehan B and Rak J: Microvesicles: Messengers and mediators of tumor progression. Cell Cycle 8: 2014-2018, 2009

43. Wendler F, Favicchio R, Simon T, Alifrangis C, Stebbing J and Giamas G: Extracellular vesicles swarm the cancer microenvironment: From tumor-stroma communication to drug intervention. Oncogene 36: 877-884, 2017.

44. Balaj L, Lessard R, Dai L, Cho YJ, Pomeroy SL, Breakefield XO and Skog J: Tumour microvesicles contain retrotransposon elements and amplified oncogene sequences. Nat Commun 2: 180, 2011.

45. Qu JL, Qu XJ, Zhao MF, Teng YE, Zhang Y, Hou KZ, Jiang YH, Yang XH and Liu YP: Gastric cancer exosomes promote tumour cell proliferation through PI3K/Akt and MAPK/ERK activation. Dig Liver Dis 41: 875-880, 2009.
46. Salnikov AV, Liu L, Platen M, Gladkich J, Salnikova O, Ryschich E, Mattern J, Moldenhauer G, Werner J, Schemmer P, et al: Hypoxia induces EMT in low and highly aggressive pancreatic tumor cells but only cells with cancer stem cell characteristics acquire pronounced migratory potential. PLoS One 7: e46391, 2012.

47. Zhou Y, Xiong M, Fang L, Jiang L, Wen P, Dai C, Zhang CY and Yang J: miR-21-containing microvesicles from injured tubular epithelial cells promote tubular phenotype transition by targeting PTEN protein. Am J Pathol 183: 1183-1196, 2013.

48. Kim J, Kim TY, Lee MS, Mun JY, Ihm C and Kim SA: Exosome cargo reflects TGF- $\beta 1$-mediated epithelial-to-mesenchymal transition (EMT) status in A549 human lung adenocarcinoma cells. Biochem Biophys Res Commun 478: 643-648, 2016.

49. Ribatti D, Mangialardi G and Vacca A: Stephen Paget and the 'seed and soil' theory of metastatic dissemination. Clin Exp Med 6: 145-149, 2006.

50. Witz IP: The tumor microenvironment: The making of a paradigm. Cancer Microenviron 2 (Suppl 1): 9-17, 2009.

51. Wiig H, Tenstad O, Iversen PO, Kalluri R and Bjerkvig R: Interstitial fluid: The overlooked component of the tumor microenvironment? Fibrogenesis Tissue Repair 3: 12, 2010.

52. Chouaib S, Kieda C, Benlalam H, Noman MZ, Mami-Chouaib F and Rüegg C: Endothelial cells as key determinants of the tumor microenvironment: Interaction with tumor cells, extracellular matrix and immune killer cells. Crit Rev Immunol 30: 529-545, 2010.

53. Vlodavsky I, Korner G, Ishai-Michaeli R, Bashkin P, Bar-Shavit R and Fuks Z: Extracellular matrix-resident growth factors and enzymes: Possible involvement in tumor metastasis and angiogenesis. Cancer Metastasis Rev 9: 203-226, 1990.

54. Micke P and Ostman A: Exploring the tumour environment: Cancer-associated fibroblasts as targets in cancer therapy. Expert Opin Ther Targets 9: 1217-1233, 2005.

55. Cho JA, Park H, Lim EH and Lee KW: Exosomes from breast cancer cells can convert adipose tissue-derived mesenchymal stem cells into myofibroblast-like cells. Int J Oncol 40: 130-138, 2012.

56. Dings R, Vang K, Griffioen A, Farrar M and Mayo K: Angiogenesis inhibitors promote T-cell mediated anti-tumor response by diminishing tumor counter immuno-surveillance. Cancer Res 68: 1119, 2008.

57. Caillou B, Talbot M, Weyemi U, Pioche-Durieu C, Al Ghuzlan A, Bidart JM, Chouaib S, Schlumberger $M$ and Dupuy C: Tumor-associated macrophages (TAMs) form an interconnected cellular supportive network in anaplastic thyroid carcinoma. PLoS One 6: e22567, 2011.

58. Yang M, Chen J, Su F, Yu B, Su F, Lin L, Liu Y, Huang JD and Song E: Microvesicles secreted by macrophages shuttle invasion-potentiating microRNAs into breast cancer cells. Mol Cancer 10: 117, 2011.

59. Ying $X, W u$ Q, Wu X, Zhu Q, Wang X, Jiang L, Chen X and Wang $X$ : Epithelial ovarian cancer-secreted exosomal miR-222-3p induces polarization of tumor-associated macrophages. Oncotarget 7: 43076-43087, 2016

60. Hsieh CH, Tai SK and Yang MH: Snail-overexpressing Cancer Cells Promote M2-Like Polarization of Tumor-Associated Macrophages by Delivering MiR-21-Abundant Exosomes. Neoplasia 20: 775-788, 2018.

61. Huang Q, Duan L, Qian X, Fan J, Lv Z, Zhang X, Han J, Wu F, Guo M, Hu G, et al: IL-17 Promotes Angiogenic Factors IL-6, IL-8, and Vegf Production via Stat1 in Lung Adenocarcinoma. Sci Rep 6: 36551, 2016.

62. Szajnik M, Czystowska M, Szczepanski MJ, Mandapathil M and Whiteside TL: Tumor-derived microvesicles induce, expand and up-regulate biological activities of human regulatory T cells (Treg). PLoS One 5: e11469, 2010.

63. Blonska M, Agarwal NK and Vega F: Shaping of the Tumor Microenvironment: Stromal Cells and Vessels. Semin Cancer Biol 34: 3-13, 2015.

64. Atala A: Re: Microvesicles released from human renal cancer stem cells stimulate angiogenesis and formation of lung premetastatic niche. J Urol 187: 1506-1507, 2012.

65. Martins VR, Dias MS and Hainaut P: Tumor-cell-derived microvesicles as carriers of molecular information in cancer. Curr Opin Oncol 25: 66-75, 2013.

66. Alipoor SD, Mortaz E, Varahram M, Movassaghi M, Kraneveld AD, Garssen J and Adcock IM: The Potential Biomarkers and Immunological Effects of Tumor-Derived Exosomes in Lung Cancer. Front Immunol 9: 819, 2018. 
67. Heinrich LF, Andersen DK, Cleasby ME and Lawson C: Long-term high fat feeding of rats results in increased numbers of circulating microvesicles with pro-inflammatory effects on endothelial cells. Br J Nutr 113: 1704-1711, 2015.

68. Aguado BA, Bushnell GG, Rao SS, Jeruss JS and Shea LD: Engineering the pre-metastatic niche. Nat Biomed Eng 1: 1-28, 2017.

69. Hood JL, San RS and Wickline SA: Exosomes released by melanoma cells prepare sentinel lymph nodes for tumor metastasis. Cancer Res 71: 3792-3801, 2011.

70. Melo SA, Luecke LB, Kahlert C, Fernandez AF, Gammon ST, Kaye J, LeBleu VS, Mittendorf EA, Weitz J, Rahbari N, et al: Glypican-1 identifies cancer exosomes and detects early pancreatic cancer. Nature 523: 177-182, 2015.

71. Semenza GL: The hypoxic tumor microenvironment: A driving force for breast cancer progression. Biochim Biophys Acta 1863: 382-391, 2016

72. Svensson K: Mechanistic Studies On the Role of Polyamines and Microvesicles in Tumor Growth and Hypoxia-mediated Angiogenesis. Scand J Dent Res 80: 139-154, 2012.

73. Lima LG, Chammas R, Monteiro RQ, Moreira ME and Barcinski MA: Tumor-derived microvesicles modulate the establishment of metastatic melanoma in a phosphatidylserine-dependent manner. Cancer Lett 283: 168-175, 2009.

74. Mineo M, Garfield SH, Taverna S, Flugy A, De Leo G, Alessandro R and Kohn EC: Exosomes released by K562 chronic myeloid leukemia cells promote angiogenesis in a Src-dependent fashion. Angiogenesis 15: 33-45, 2012.

75. Rahbari M, Rahbari N, Reissfelder C, Weitz J and Kahlert C: Exosomes: Novel implications in diagnosis and treatment of gastrointestinal cancer. Langenbecks Arch Surg 401: 1097-1110, 2016.

76. Diaz LA Jr and Bardelli A: Liquid biopsies: Genotyping circulating tumor DNA. J Clin Oncol 32: 579-586, 2014.

77. Armstrong D and Wildman DE: Extracellular Vesicles and the Promise of Continuous Liquid Biopsies. J Pathol Transl Med 52: $1-8,2018$

78. Wieckowski EU, Kim JW, Stanson JD, Taylor DD, Reichert TE and Whiteside TL: FasL+ and FasL- microvesicles in the circulation of patients with cancer induce apoptosis of activated T lymphocytes. Cancer Res 64: Issue 7, 2004.

79. Hosseinibeheshti E, Pham S, Adomat H, Li N and Tomlinson Guns ES: Exosomes as biomarker enriched microvesicles: characterization of exosomal proteins derived from a panel of prostate cell lines with distinct AR phenotypes. Mol Cell Proteomics 11: 863-885, 2012.

80. Baran J, Baj-Krzyworzeka M, Weglarczyk K, Szatanek R, Zembala M, Barbasz J, Czupryna A, Szczepanik A and Zembala M: Circulating tumour-derived microvesicles in plasma of gastric cancer patients. Cancer Immunol Immunother 59: 841-850, 2010

81. Valenti R, Huber V, Filipazzi P, Pilla L, Sovena G, Villa A, Corbelli A, Fais S, Parmiani G and Rivoltini L: Human tumor-released microvesicles promote the differentiation of myeloid cells with transforming growth factor-beta-mediated suppressive activity on T lymphocytes. Cancer Res 66: 9290-9298, 2006.

82. Kimura S, Naganuma S, Susuki D, Hirono Y, Yamaguchi A, Fujieda S, Sano K and Itoh H: Expression of microRNAs in squamous cell carcinoma of human head and neck and the esophagus: miR-205 and miR-21 are specific markers for HNSCC and ESCC. Oncol Rep 23: 1625-1633, 2010.
83. Kachakova D, Mitkova A, Popov E, Popov I, Vlahova A, Dikov T, Christova S, Mitev V, Slavov C and Kaneva R: Combinations of serum prostate-specific antigen and plasma expression levels of let-7c, miR-30c, miR-141, and miR-375 as potential better diagnostic biomarkers for prostate cancer. DNA Cell Biol 34: 189-200, 2015

84. Kahlert C, Melo SA, Protopopov A, Tang J, Seth S, Koch M, Zhang J, Weitz J, Chin L, Futreal A, et al: Identification of double-stranded genomic DNA spanning all chromosomes with mutated KRAS and p53 DNA in the serum exosomes of patients with pancreatic cancer. J Biol Chem 289: 3869-3875, 2014.

85. Borecka M, Zemankova P, Vocka M, Soucek P, Soukupova J, Kleiblova P, Sevcik J, Kleibl Z and Janatova M: Mutation analysis of the PALB2 gene in unselected pancreatic cancer patients in the Czech Republic. Cancer Genet 209: 199-204, 2016.

86. Vader P, Breakefield XO and Wood MJ: Extracellular vesicles: Emerging targets for cancer therapy. Trends Mol Med 20: 385-393, 2014

87. Sidhu SS, Mengistab AT, Tauscher AN, LaVail J and Basbaum C: The microvesicle as a vehicle for EMMPRIN in tumor-stromal interactions. Oncogene 23: 956-963, 2004.

88. Jorfi $\mathrm{S}$ and Inal JM: The role of microvesicles in cancer progression and drug resistance. Biochem Soc Trans 41: 293-298, 2013.

89. Chen WX, Zhong SL, Ji MH, Pan M, Hu Q, Lv MM, Luo Z, Zhao JH and Tang JH: MicroRNAs delivered by extracellular vesicles: An emerging resistance mechanism for breast cancer. Tumour Biol 35: 2883-2892, 2014.

90. Esteva FJ: Novel strategies for HER-2-positive metastatic disease: Mechanisms and therapeutic options to overcome trastuzumab resistance. Breast Cancer Res 9 (Suppl 1): 1-2, 2007.

91. Silva AK, Luciani N, Gazeau F, Aubertin K, Bonneau S, Chauvierre C, Letourneur D and Wilhelm C: Combining magnetic nanoparticles with cell derived microvesicles for drug loading and targeting. Nanomedicine 11: 645-655, 2015

92. Vader P, Mol EA, Pasterkamp G and Schiffelers RM Extracellular vesicles for drug delivery. Adv Drug Deliv Rev 106 (Pt A): 148-156, 2016

93. Tian Y, Li S, Song J, Ji T, Zhu M, Anderson GJ, Wei J and Nie G: A doxorubicin delivery platform using engineered natural membrane vesicle exosomes for targeted tumor therapy. Biomaterials 35: 2383-2390, 2014.

94. Mizrak A, Bolukbasi MF, Ozdener GB, Brenner GJ, Madlener S, Erkan EP, Ströbel T, Breakefield XO and Saydam O: Genetically engineered microvesicles carrying suicide mRNA/protein inhibit schwannoma tumor growth. Mol Ther 21: 101-108, 2013.

95. Kim HO, Choi SM and Kim HS: Mesenchymal stem cell-derived secretome and microvesicles as a cell-free therapeutics for neurodegenerative disorders. Tissue Eng Regen Med 10: 93-101, 2013.

96. Miller VM, Lahr BD, Bailey KR, Hodis HN, Mulvagh SL and Jayachandran M: Specific cell-derived microvesicles: Linking endothelial function to carotid artery intima-media thickness in low cardiovascular risk menopausal women. Atherosclerosis 246: 21-28, 2016.

97. Kahlert C and Kalluri R: Exosomes in tumor microenvironment influence cancer progression and metastasis. J Mol Med (Berl) 91: 431-437, 2013. 